\title{
Different Therapeutical Attitudes against Enuresis the Prosecution of a Clinical Experience
}

\author{
Giorgio Bolla*, Gianluca Sartore \\ Department of Paediatric Urology - Public Hospital Dolo (Venice) - Italy \\ *Corresponding author: giorgio.bolla@libero.it
}

Received February 01, 2014; Revised April 08, 2014; Accepted April 15, 2014

\begin{abstract}
The differentiation of therapies for nocturnal enuresis responds to peculiarities of children and their families. In this study three modalities of approach to enuretic symptom were evaluated: the sole behavioral approach, the alarm treatment and the use of desmopressin; we have envisaged the response to treatment for 90 children. In particular, the effectiveness of desmopressin suggests that the cause of monosymptomatic nocturnal enuresis often lies in the structure of the sleep.
\end{abstract}

Keywords: nocturnal enuresis, desmopressin

Cite This Article: Giorgio Bolla, and Gianluca Sartore, "Different Therapeutical Attitudes against Enuresis the Prosecution of a Clinical Experience.” American Journal of Clinical Medicine Research, vol. 2, no. 2 (2014): 51-52. doi: 10.12691/ajcmr-2-2-3.

\section{Introduction}

Enuresis: functional anomaly or else clinical picture manageable by specific therapeutic approaches [1]? The classical definition of monosymptomatic nocturnal enuresis assumes a clinical situation without daytime incontinence.

Nocturnal enuresis results when the bladder is able to fill to its functional capacity and contract reflexively during sleep. While nocturnal enuresis is normal in infants, a series of maturational processes, hormonal, neural and structural, results in nocturnal urinary continence by age 5 years in most children. Maturational delay or pathological alteration in one or more of these processes can result in persistent primary enuresis or secondary onset of nocturnal enuresis. However, the tendency to spontaneous resolution of enuresis is equal to $15 \%$ per annum $[2,3]$.

The Authors intended to verify whether the differentiation of therapies can influence the clinical outcome.

\section{Methodology}

From April 2008 to September 2012 a total of 90 children suffering from nocturnal enuresis - 25,5\% of children with persistent primary enuresis - underwent a different treatment: 30 children were treated with a behavioral approach, 30 children by alarm treatment and 30 children with desmopressin. The interval of age was 5 y. $1 \mathrm{~m} .-12$ y. 7 m., while there was a light prevalence for the male sex (49 vs 41). All children received a directed physical and neurological examination and underwent at least one analysis of micturition day-books. Urinary tract imaging with ultrasound studies was performed. We obtained the informed consent from the parents and the ethics approval according to Helsinki Declaration. The selection for different groups of therapy was achieving with random pattern; afterwards, the presence in the patients of a sound sleep with a difficult arousal and/or nocturnal polyuria allowed us to prefer for them the employment of desmopressin.

The behavioral instructions regarded the timing of drinking and the management of voiding. The acoustic alarms in use were belonging to the rising generation of instruments, while the drug desmopressin was employed for three months - $120 \mu \mathrm{g} /$ night, below the tongue -; in case of defective outcome of the pharmacologic treatment - 6/30, 20\% of children - we have repeated the administration with same modalities, ever for three months. The definition of therapeutic success was relative to the absence of monosymptomatic enuresis for 14 following nights, without relapses. Mean follow-up was for the first group $15 \mathrm{~m}$. and $9 \mathrm{~d}$., for the second $16 \mathrm{~m}$. and $5 \mathrm{~d}$., for the third $18 \mathrm{~m}$. and $16 \mathrm{~d}$..

The statistical analysis about the results of treatments was performing by the Wilcoxon Test for independent samples (N1 30 - N2 30 - N3 30).

\section{Results}

In 29 (96.6\%) of children treated with desmopressin the drug resolved their illness, while $10 \%$ of them treated by alarm and $16.6 \%$ of them treated with behavioral intervention persisted in symptom of enuresis (Figure 1). In pretreatment values the circadian rhythm of urine production and the urine osmolality were similar between full responders and nonfull responders of the second and the third group of treatment. No one of 30 children treated 
with desmopressin lamented for unfavourable effects by the therapy: only four children (13.3\%) treated with the drug have developed a temporary asymptomatic hyponatraemia; moreover, we didn’t find urinary tract infections.

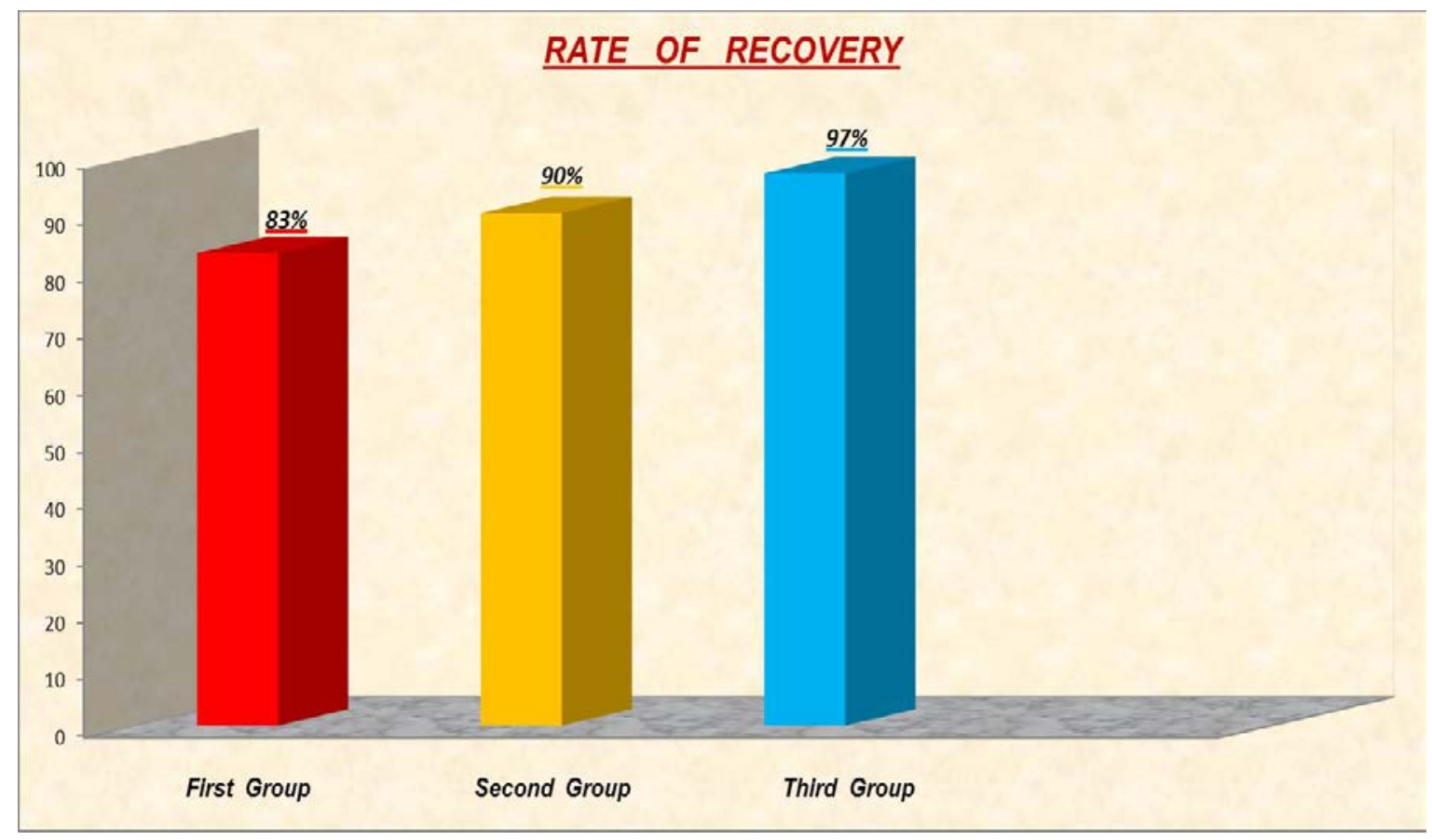

Figure 1. Rate of Recovery into the different approaches to Enuresis (see text)

\section{Discussion}

The clinical samples, in our experience, were too little for a statistical significance, however the trend about the results of different treatments had been sufficiently definite, in favour of the group treated with desmopressin.

The clinical remarks point out the coexistence of two categories of enuretic children:

A) Children with anomalous rhythm between sleeping and waking.

B) Children with prevalence of detrusor instability (the only child who failed into the group treated with desmopressin showed a very important instability by an urodynamic study: this investigation, achieved after the second useless therapeutic approach with desmopressin, took us to an effective employment of anticholinergic).

On the contrary, a pattern of nocturnal enuresis secondly noticed gives evidence against the delay of vesico-sphincteric maturation $[4,5]$.

The employment of desmopressin follows the hypothesis of anomalous nocturnal secretion of the antidiuretic hormone - AVP -; however, we think this causal condition frequent but not sole [6,7].

We believe in the necessity of differentiation of treatments, as regards the cultural level of the domestic environment and the psychologic pattern of child [8].

\section{Conclusion}

The clear awareness of the problem frequently leads a child and his own family to the recovery from the enuresis with sole behavioral intervention.

In case of a learned environment the use of acoustic alarm is very effective.
The pharmacologic treatment, wanting in unfavourable effects, takes significance when there is need to resolve the problem of nocturnal enuresis rapidly - for instance in case of community journeys.

\section{Conflict of Interest}

None

\section{References}

[1] Bolla G., Sartore G. Children with nocturnal enuresis: why several treatments? (in Italian). Med. Surg. Ped. 2010; 179: 226-228.

[2] Hjalmas K., Arnold T,. Bower W., et al. Nocturnal enuresis: an international evidence based management strategy. J. Urol. 2004; 171: 2545-2561.

[3] Yeung C.K., Sihoe J.D.Y., Sit F.K.Y., et al. Urodynamic findings in adult with primary nocturnal enuresis. J. Urol. 2004; 171: 25952598.

[4] Chandra M., Saharia R., Hill V., et al. Prevalence of diurnal voiding symptoms and difficult arousal from sleep in children with nocturnal enuresis. J. Urol. 2004; 172: 311-316.

[5] Dehoorne J.L.C., Vande Walle C., Vansintjan P., et al. Characteristics of a tertiary center enuresis population, with special emphasis on the relation among nocturnal diuresis, functional bladder capacity and desmopressin response. J. Urol. 2007; 177: 1130-1137.

[6] Kamperis K., Hansen M.N., Hagstroem S., et al. The circadian rhythm of urine production, and urinary vasopressin and prostaglandin E2 excretion in healthy children. J. Urol. 2004; 171: 2571-2575.

[7] Akbal C., Ekici S., Erkan I., et al. Intermittent oral desmopressin therapy for monosymptomatic primary nocturnal enuresis. J. Urol. 2004; 171; 2603-2606.

[8] Van Kampen M., Bogaert G., Akinwuntan E.A., et al. Long-term efficacy and predictive factors of full spectrum therapy for nocturnal enuresis. J. Urol. 2004; 171: 2599-2602. 